\title{
ENIGMAS FROM THE SLOAN DIGITAL SKY SURVEY DR7 KLEINMAN WHITE DWARF CATALOG
}

\author{
James Liebert $^{1}$, Lilia Ferrario ${ }^{2}$, Dayal T. Wickramasinghe ${ }^{2}$, and Paul S. Smith ${ }^{1}$ \\ ${ }^{1}$ Steward Observatory, University of Arizona, Tucson, AZ 85721, USA; jamesliebert@gmail.com, psmith@as.arizona.edu \\ ${ }^{2}$ Mathematical Sciences Institute, Australian National University, ACT 0200, Australia; Lilia.Ferrario@anu.edu.au, Dayal.Wickramasinghe@anu.edu.au \\ Received 2014 December 1; accepted 2015 February 24; published 2015 May 6
}

\begin{abstract}
We report results from a continuation of our searches for high field magnetic white dwarfs (WDs) paired in a detached binary with non-degenerate companions. We made use of the Sloan Digital Sky Survey DR7 catalog of Kleinman et al. (2013) with 19,712 spectroscopically identified WDs. These include 1735 WD plus M dwarf detached pairs (almost 10\% of the Kleinman et al.'s list). No new pairs were found, although we did recover the polar (AM Herculis system) ST LMi in a low state of accretion. With the larger sample the original situation reported $10 \mathrm{yr}$ ago remains intact now at a much higher level of statistical significance: in the selected SDSS sample, high field magnetic WDs are not found in an apparently detached pairing with an M dwarf, unless they are a magnetic cataclysmic variable (CV) in a low state of accretion. This finding strengthens the case that the fields in the isolated high field magnetic WDs are generated by stellar mergers but also raises questions on the nature of the progenitors of the magnetic CVs.
\end{abstract}

Key words: binaries: general - polarization - stars: evolution - stars: individual (STLMi, SDSS110539.768 $+250628.62)$ - white dwarfs

\section{INTRODUCTION}

After Data Release 1 (2004) of the Sloan Digital Sky Survey (York et al. 2000), some 169 magnetic white dwarfs (WDs) were known with fields greater than about 2 mega-Gauss (MG) from SDSS and other sources. We refer to these high field magnetic WDs as MagWDs. In the same release, some 501 detached binary systems consisting of a WD and nondegenerate companion were listed. The companion was nearly always an M dwarf. Some $10 \mathrm{yr}$ ago Liebert et al. (2005) noticed a curious fact: the two subsets of MagWDs and detached WD + dM's did not intersect. To confirm this, we visually inspected all MagWDs and WD + dM's. Indeed, no MagWD had a non-degenerate companion, and no WD + dM detached pairs included a MagWD. These results were surprising, considering the fact that some $8 \%$ of isolated WDs were MagWDs (Wickramasinghe \& Ferrario 2000) and led Tout et al. (2008) to propose that the isolated MagWDs were the result of a stellar merger where the fields were generated during the merger process, a proposal that has since gained considerable momentum (Nordhaus et al. 2011; GarciaBerro et al. 2012; Wickramasinghe et al. 2014; Briggs et al. 2015).

The SDSS WD + detached M samples include both wide pairs in which the progenitor of the WD and the $\mathrm{dM}$ were evolved without interaction and pairs that underwent common envelope evolution-the so-called post common envelope binaries (PCEBs). The latter group will evolve into the cataclysmic variables (CVs), semi-detached close binary systems where a non-degenerate late-type companion transfers matter to a WD. Now about one quarter of known CVs have WDs with fields in the range $\sim 1-1000 \mathrm{MG}$, the so-called magnetic CVs (MagCVs, see the review by Wickramasinghe \& Ferrario 2000). Our failure to detect magnetic fields in the detached $\mathrm{WD}+\mathrm{dM}$ sample therefore also raises the question "where are the PCEBs that are the progenitors of the MagCVs?"

\section{THE CURRENT STUDY}

In this paper, we report on the continuation of the same searches utilizing the much larger Data Release 7 (DR7) of SDSS. Kleinman et al. (2013) have compiled a WD catalog, with some 19,712 spectroscopically identified WDs with SDSS spectra. They also visually identified 804 MagWDs. Kepler et al. (2013) also visually inspected the SDSS spectra and estimated the field strength and masses of 521 DA MagWDs. Their work has significantly increased the number of known MagWDs. However, none of them were reported to have a companion.

We visually inspected all $1735 \mathrm{WD}+\mathrm{dM}$ pairs again (1951 individual spectra including duplicates), almost $10 \%$ of the WD sample listed by Kleinman et al. (2013). Most of these binaries are also cataloged in Rebassa-Mansergas et al. (2010, 2012). Our search was motivated by our knowledge that it would have been easy to miss recognizing the presence of a magnetic field in a WD in previous such studies, particularly if the field is either very high, or the field structure is peculiar, or the spectrum is due to elements other than hydrogen. The discovery of such a system would have required follow-up polarimetric or spectropolarimetric observations for confirmation, but none was found.

We thought that we had found one case where a moderate field MagWD might be paired with an M dwarf in a detached binary. The SDSS object, designated SDSSJ 110539.77+250628.5, appeared to be a detached binary system with a probable magnetic WD having an dM5-6 secondary companion. Despite the high signal-to-noise ratio $(\mathrm{S} / \mathrm{N})$ of the spectrum, the probable magnetic features did not appear as simple Zeeman splitting of hydrogen lines. The $\mathrm{H} \beta$ transition was clearly present in absorption, but a clear Zeeman triplet was not obvious. A narrow $\mathrm{H} \alpha$ emission line, presumably coming from the late-M secondary, was also present in the spectrum. To confirm its magnetic nature polarimetric observations were obtained on several separate runs in 2014 June (by PSS) on the Catalina Observatory $1.55 \mathrm{~m}$ with the SPOL 
spectropolarimeter. These showed that the object was an AM Herculis system (polar) in a high state of accretion. It turned out that the low state SDSS spectrum that had led us to believe that this was a detached system, had already been published by Szkody et al. (2009) in a paper on multiple CVs observed in the SDSS. Here the system had been identified as the well known Polar STLMi with an orbital period of $P_{\text {orb }}$ $=113.9$ minutes and a field $B=12 \mathrm{MG}$ (Ferrario et al. 1993) in a very low state of accretion. The spectrum that we obtained was similar to that obtained by Stockman et al. (1983). During the orbital period, there is a $\sim 40$ minutes "bright phase" when the actively accreting pole is in view, and the circular polarization is about $15 \%$. There is a $\sim 74$ minute "faint phase" where the circular polarization is essentially zero (when the active pole is out of view). A cyclotron harmonic accounts for the rise in the spectrum at longer wavelengths, when the bright pole is in view. We have added nothing new to the understanding of ST LMi, other than that its behavior has not changed in the last $\sim 30 \mathrm{yr}$.

The system we had found was clearly not what we were originally looking for, that is, a detached MagWD + M dwarf system.

\section{DISCUSSION AND CONCLUSIONS}

Our visual search for apparently detached WD + dM's from the SDSS DR7 leads us to the conclusion that there is still no known MagWD paired with a non-degenerate companion. The sample size for these searches is now about four times as large as it was $10 \mathrm{yr}$ ago, approaching a total of 20,000 objects. A simple $\chi^{2}$ test assuming one degree of freedom shows that the hypothesis that magnetic field and binarity (when paired with $\mathrm{K}$ or $\mathrm{M}$ dwarfs) are independent is now vindicated at the $9 \sigma$ level. Notwithstanding the fact that WDs with super-strong fields particularly in spectra of low $\mathrm{S} / \mathrm{N}$ are difficult to identify so that some potential candidates may have been missed, the case is now overwhelming that there is essentially no intersection between MagWDs and WD $+d M$ detached pairs.

The continued absence of detached MagWD and non-degenerate dwarf pairings reinforces the ideas of Regös and Tout (1995), Tout et al. (2008), and Wickramasinghe et al. (2014) that strong fields in isolated MagWDs are generated by a dynamo mechanism that feeds on the differential rotation that occurs either during common envelope evolution of a binary system that results in a merger or during a double degenerate merger (see also Nordhaus et al. 2011; Garcia-Berro et al. 2012). Theory suggests that a variety of magnetic field strengths can result from such mergers, with the field strength perhaps increasing with the combined mass (see, e.g., Tout et al. 2008). Evidence that the mass increases with MagWD field strength is discussed in Kepler et al. (2013). That nearby, highly magnetic WDs with accurate trigonometric parallaxes are more massive than their non-magnetic counterparts was argued by Liebert (1988).

We now turn to the CVs. Szkody et al. (2009) have identified $285 \mathrm{CV}$ s from the SDSS DR7 release and about $20 \%$ of these are magnetic. Our failure to detect detached systems with a MagWD again raises the question "where are the PCEBs that are the progenitors of the MagCVs?"

There have been specific searches for PCEBs using SDSS surveys augmented by radial velocity measurements (e.g., Rebassa-Mansergas et al. 2010, 2012; Zorotovic et al. 2011; Parsons et al. 2013a). These studies have shown that roughly one-third of the detached WD + dM pairs in SDSS are PCEBs. Zorotovic et al. (2011) listed some 60 PCEBs with well determined orbital periods and WD effective temperatures. The periods range from 0.08 to 20 days and effective temperatures from $\sim 7500-60,000 \mathrm{~K}$. Magnetic braking and gravitational radiation will lose angular momentum from the orbit and bring about a third of these systems into contact within a Hubble time. Interestingly, none of these had MagWD companions.

There is a known class of binary system whose members could be identified as PCEB pre-polars. These were initially referred to as LARPs-Low Accretion Rate Polars (Reimers et al. 1999; Reimers \& Hagen 2000; Schwope et al. 2002; Schmidt et al. 2005a, 2007). They are detached binaries in which the MagWD accretes matter at a very low rate from a stellar wind that emanates from the non-degenerate companion (Webbink \& Wickramasinghe 2005). The magnetic nature of the LARPs was discovered through the detection of cyclotron emission humps in their energy distribution from accretion shocks on the WD surface that were superposed on the WD + dM stellar continuum (e.g., Ferrario et al. 2005). Such systems are not expected to be picked up in surveys such as the one used by us to select normally looking WD + main sequence stellar continua. In fact the first such systems were discovered in surveys aimed at discovering active galactic nuclei!

Schwope et al. (2009) have summarized the properties of the nine known LARPs, which were renamed as PREPs (prepolars) to avoid confusion with Polars in low states of accretion. These systems have short orbital periods very similar to but on average marginally larger that those of Polars. Their WDs have temperatures that are typically much less than $10,000 \mathrm{~K}$, and their cooling ages are estimated to be greater than about 200 million years with most having ages of above a billion years. In fact, the WDs in Roche lobe-filling polars tend to have somewhat higher tempertures with the difference attributed to different amount of accretion heating in the high and low modes of accretion. Schmidt et al. (2005a) argued that there were field biases in the PREPs discovered from SDSS that depended on the strength and positioning of cyclotron harmonics in the filter bands used in the selection process that favored only the discovery of strong field $(\sim 40-100 \mathrm{MG})$ systems. It is reasonable to expect larger numbers of lower field systems in similar states of accretion, although they would be less easy to detect as the cyclotron emission peak moves further into the infrared (see Parsons et al. 2013b).

In addition to PREPs discovered through the detection of cyctrotron lines, there are also MagWDs such as SDSSJ $121209.31+013627.7$ (Schmidt et al. 2005b; Burleigh et al. 2006; Debes et al. 2006; Koen \& Maxted 2006; Farihi et al. 2008; Linnell et al. 2010) that appear single in SDSS spectra but show the presence of very low mass companions when observed further in the infrared (Breedt et al. 2012). Although in many of these cases it is unclear if they represent PREPs or MagCVs in an extended low state, it is likely that some of these systems are also PREPs.

The known PREPs, due to their low effective temperatures and large cooling ages, are clearly in a very late stage of post common envelope evolution. The hotter PREPs (and more generally MagCVs) should be found among the PCEBs extracted from SDSS and other sources (Zorotovic et al. 2011) which does have many hot WDs. However, although some $20 \%$ of known CVs are magnetic, their birthrate 
may be significantly smaller that that of non-magnetic CVs because of the expected reduction in magnetic braking in these systems (Li et al. 1994; Webbink \& Wickramasinghe 2002) for which there is some observational evidence (Araujo-Betancor et al. 2005; Townsley and Gänsicke 2009). The absence of hot PREPs may not be surprising given the small sample size, and with better statistics it may indeed turn out that the PREPs can be identified as the progenitors of the MagCVs as was already suggested by Webbink \& Wickramasinghe (2002).

We conclude by noting that the physics of the common envelope phase of evolution is poorly understood and, not surprisingly, there are no detailed studies of binary evolution that allow for the generation of magnetic fields during the common envelope phase. A prediction of the Tout et al. (2008) model is that the magnetic fields of the WDs are generated during the common envelope phase with systems that merge producing isolated MagWDs and systems that do not merge, but come closer to contact, being the progenitors of the MagCVs (Polars and Intermediate Polars). As our present study has shown, the stellar merging hypothesis appears to be consistent with all available observations and has also been supported by recent population synthesis studies (Briggs et al. 2015). The question of why the isolated high field MagWDs on the one hand, and the Polars and Intermediate Polars on the other, both emerge from binary evolution with magnetic fields in the same range (1-1000 MG) could be related to the robustness of the dynamo model that invokes differential rotation for the generation of fields (Wickramasinghe et al. 2014). These aspects of the model should be testable by observations when a larger sample of PCEBs that all include pre-MagCVs becomes available.

We thank Boris Gänsicke for drawing our attention to the catalog of CVs based on SDSS where the low state spectrum of STLMi had already been reported. We also thank Scot Kleinman and the referee for helpful suggestions.

\section{REFERENCES}

Araujo-Betancor, S., Gänsicke, B. T., Long, K. S., et al. 2005, ApJ, 622, 598 Breedt, E., Gänsicke, B. T., Girven, J., et al. 2012, MNRAS, 423, 1437

Briggs, G., Ferrario, L., Tout, C. A., Wickramasinghe, D. T., \& Hurley, J. 2015, MNRAS, 447, 1713

Burleigh, M. R., Marsh, T. R., Gänsicke, B. T., et al. 2006, MNRAS, 373,1416
Debes, J. H., López-Morales, M., Bonanos, A. Z., \& Weinberger, A. J. 2006, ApJ, 547, 147

Farihi, J., Burleigh, M. R., \& Hoard, D. W. 2008, ApJ, 674, 421

Ferrario, L., Bailey, J. A., \& Wickramasinghe, D. T. 1993, MNRAS, 262, 285

Ferrario, L., Wickramasinghe, D. T., \& Schmidt, G. D. 2005, in Proc. ASP Conf. 330, The Astrophysics of Cataclysmic Variables and Related Objects, ed. J.-M. Hameury, \& J.-P. Lasota (San Francisco: ASP), 411

Garcia-Berro, E., Lorén-Aguilar, R., Aznar-Siguān, G., et al. 2012, ApJ, 749,25

Kepler, S. O., Pelisoli, I., Jordan, S., et al. 2013, MNRAS, 429, 2934

Kleinman, S. J., Kepler, S. O., Koester, D., et al. 2013, ApJS, 204, 5

Koen, C., \& Maxted, P. F. L. 2006, MNRAS, 371, 1675

Li, J., Wu, K., \& Wickramasinghe, D. T. 1994, MNRAS, 270, 769

Liebert, J. 1988, PASP, 100, 1302

Liebert, J., Wickramasinghe, D. T., Schmidt, G. D., et al. 2005, AJ, 129, 2376

Linnell, A. P., Szkody, P., Plotkin, R. M. H. J., et al. 2010, ApJ, 713, 1183

Nordhaus, J., Wellons, S., Spiegel, D. S., Metzger, B. D., \& Blackman, E. G. 2011, PNAS, 108, 3135

Parsons, S. G., Gänsicke, B. T., Marsh, T. R., et al. 2013a, MNRAS, 429, 256

Parsons, S. G., Marsh, T. R., Gänsicke, B. T., et al. 2013b, MNRAS, 436, 241

Rebassa-Mansergas, A., Gänsicke, B. T., Schreiber, M. R., Koester, D., \& Rodriguez-Gil, P. 2010, MNRAS, 402, 620

Rebassa-Mansergas, A., Nebot Gómez-Morán, A., Schreiber, M. R., et al. 2012, MNRAS, 419, 806

Regös, E., \& Tout, C. A. 1995, MNRAS, 273, 146

Reimers, D., \& Hagen, H.-J. 2000, A\&A, 358, L45

Reimers, D., Hagen, H.-J., \& Hopp, U. 1999, A\&A, 343, 157

Schmidt, G. D., Stockman, H. S., \& Grandi, S. A. 1983, ApJ, 271, 735

Schmidt, G. D., Szkody, P., Henden, A., et al. 2007, ApJ, 654, 521

Schmidt, G. D., Szkody, P., Vanlandingham, K. M., et al. 2005, ApJ, 630, 1037

Schmidt, G. D., Szkody, P., Silvestri, N. M., et al. 2005, ApJ, 630, 173

Schwope, A. D., Brunner, H., Hambaryan, V., \& Schwarz, R. 2002, in ASP Conf. Ser. 261, Physics of Cataclysmic Variables and Related Objects, ed. B. T. Gänsicke, K. Beuermann, \& K. Reinsch (San Francisco, CA: ASP), 102

Schwope, A. D., Gomez-Moran, A. M., Schreiber, M. R., \& Gansicke, B. T. 2009, A\&A, 500, 867

Stockman, H. S., Foltz, C. B., Schmidt, G. D., \& Tapia, S. 1983, ApJ, 271, 725

Szkody, P., Anderson, S. F., Hayden, M., et al. 2009, AJ, 137, 4011

Tout, C. A., Wickramasinghe, D. T., Liebert, J., Ferrario, L., \& Pringle, J. E. 2008, MNRAS, 387, 897

Townsley, T. M., \& Gänsicke, B. T. 2009, ApJ, 693, 2007

Webbink, R. F., \& Wickramasinghe, D. T. 2002, MNRAS, 335, 1

Webbink, R. F., \& Wickramasinghe, D. T. 2005, in Proc. ASP Conf. 330, The Astrophysics of Cataclysmic Variables and Related Objects, ed. J.-M. Hameury, \& J.-P. Lasota (San Francisco: ASP), 137

Wickramasinghe, D. T., \& Ferrario, L. 2000, PASP, 112, 873

Wickramasinghe, D. T., Tout, C. A., \& Ferrario, L. 2014, MNRAS, 437, 675

York, D. G., Adelman, J., Anderson, J. E., et al. 2000, AJ, 120, 1579

Zorotovic, M., Schreiber, M. R., \& Gänsicke, B. T. 2011, A\&A, 536, A42 